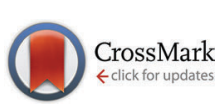

Cite this: Soft Matter, 2016, 12,998

\title{
One-step production of multiple emulsions: microfluidic, polymer-stabilized and particle-stabilized approaches
}

\begin{abstract}
Paul S. Clegg, ${ }^{\star a}$ Joe W. Tavacoli ${ }^{a}$ and Pete J. Wilde ${ }^{b}$
Multiple emulsions have great potential for application in food science as a means to reduce fat content or for controlled encapsulation and release of actives. However, neither production nor stability is straightforward. Typically, multiple emulsions are prepared via two emulsification steps and a variety of approaches have been deployed to give long-term stability. It is well known that multiple emulsions can be prepared in a single step by harnessing emulsion inversion, although the resulting emulsions are usually short lived. Recently, several contrasting methods have been demonstrated which give rise to stable multiple emulsions via one-step production processes. Here we review the current state of microfluidic, polymer-stabilized and particle-stabilized approaches; these rely on phase separation, the role of electrolyte and the trapping of solvent with particles respectively.
\end{abstract}

Received 7th July 2015,

Accepted 11th November 2015

DOI: $10.1039 / c 5 s m 01663 k$

www.rsc.org/softmatter and as a means of reducing the oil content of oil-in-water emulsion based food products. ${ }^{3-8}$ The large scale adoption of multiple emulsions has been hampered by the fact that the surfactants used are often not acceptable and that complex multi-step production routes can be required for fabrication, see Fig. 1. The first of these issues has recently been addressed in a review article; ${ }^{9}$ here we consider research directed at simplifying the fabrication route.

For the purpose of this review we define "one step" to mean emulsification using a single mixing step. The practitioner implements mixing via a high shear/high pressure approach or via the gentle pumping of one liquid into another in a microfluidic device. The advantages of one step emulsification are (1) simplicity: only one set of mixing conditions have to be

Road, Edinburgh, EH9 3FD, UK. E-mail: paul.clegg@ed.ac.uk

${ }^{b}$ Institute of Food Research, Norwich Research Park, Norwich NR4 7UA, UK

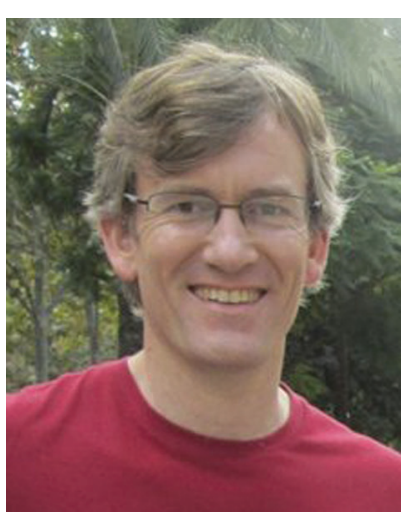

Paul S. Clegg
Paul Clegg is a reader in the Soft Matter group at the University of Edinburgh. His research focuses on the novel behaviour of soft materials which are stabilized by complex interfaces.

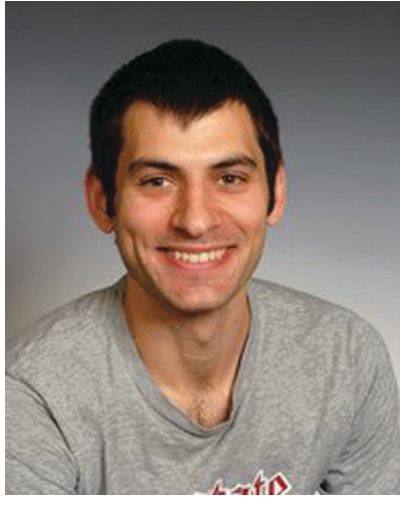

Joe Tavacoli is an industry research liaison in the Soft Matter group at the University of Edinburgh. His recent projects have spanned lipid behaviour, slow dynamics in soft matter systems and encapsulating volatile liquids.

Joe W. Tavacoli 
a
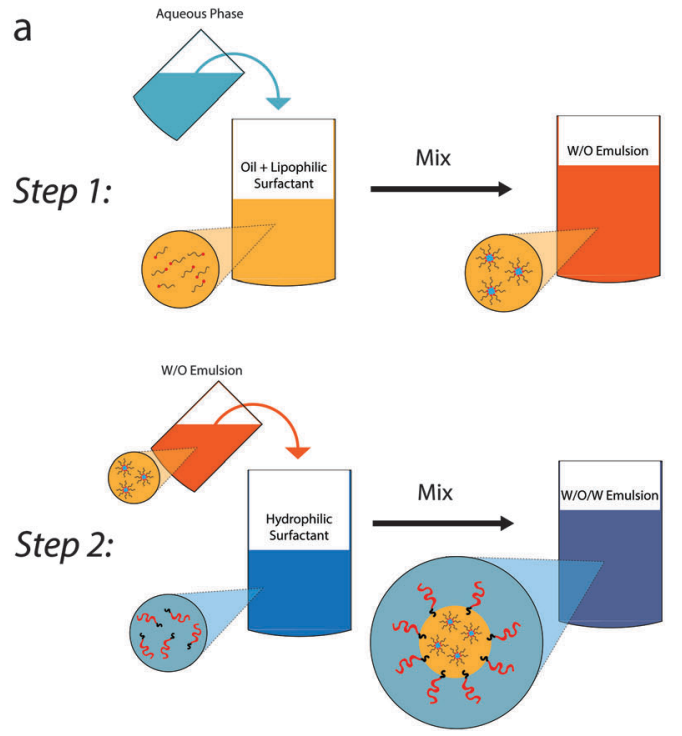

b

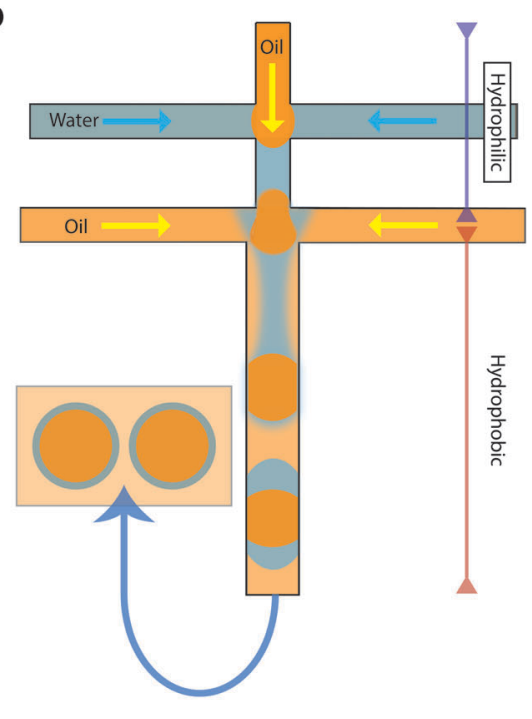

Fig. 1 Schematics showing the two step production of a multiple emulsion. (a) Conventional batch emulsification: step 1 is creating the internal droplets, step 2 creates the outer droplets using gentle shear. Adapted from ref. 1 with permission from Elsevier. (b) Step-wise production of multiple emulsions using microfluidics. Successive junctions are used to create the internal and external droplets. (This apparatus can be adapted for one-step emulsification. ${ }^{82}$ )

optimized (2) robustness: the second mixing step does not destabilize the first. For multiple emulsions to be widely adopted fabrication will need to be simple and robust. It should be noted that in application areas where a high value payload needs to be encapsulated within the internal droplets, two step mixing may be preferable so as to avoid losses into the continuous phase. Within this review our interests span multiple emulsions fabricated from a range of immiscible liquids, not only simple oil-water systems. We focus on the production process while discussing stabilization in less detail.

Multiple emulsions have often been observed to form in a single-step process close to the composition and conditions at which the emulsion inverts (the dispersed phase becoming the continuous phase and vice versa). Indeed, the first observation was made during the study of the inversion of a petroleum/ water emulsion..$^{10}$ When molecular surfactants are used for stabilization, the multiple emulsions formed close to inversion

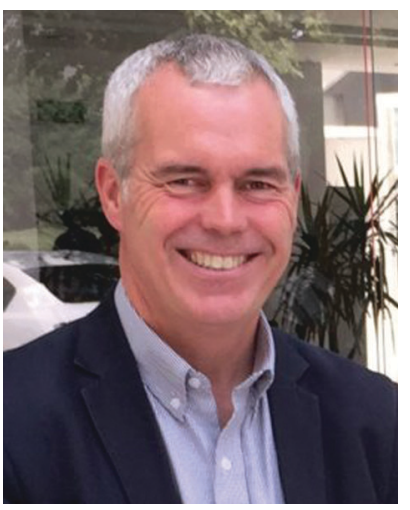

Pete J. Wilde
Pete Wilde leads the Food Structure research programme at the Institute of Food Research in Norwich. His main research interest is studying the interfacial properties of surface-active food components. are typically highly unstable. Hence this fabrication route has not been extensively exploited. Instead, stable multiple emulsions are more commonly prepared by a two-step route: the internal droplets are created first at high shear using an appropriate surfactant; subsequently, the continuous phase is added with a different surfactant while the mixture is gently stirred, see Fig. 1a. This approach is somewhat involved and furthermore it often requires significant concentrations of surfactant.

One single-step emulsification route has previously been demonstrated to exhibit long term stability, see ref. 11. In this case the system is prepared from a mixture of surfactants which are first dissolved in the oil phase. The aqueous phase is added to this when both are at elevated temperature $\left(>70{ }^{\circ} \mathrm{C}\right)$. The multiple emulsion then forms as the mixture cools to room temperature; intermediate stages may involve an ultralow interfacial tension and the formation of a microemulsion. ${ }^{12}$ Such multiple emulsions can be stable for many months but need large quantities of carefully chosen combinations of surfactants designed to yield an appropriate hydrophilic lipophilic balance (HLB) on average. ${ }^{11}$

The constraint on multiple emulsions in food applications is the need to use permitted emulsifiers at relatively low concentrations. ${ }^{13}$ Proteins are widely used to stabilise food emulsions, however their structure and general hydrophilic nature does not support the stabilisation of water-in-oil (W/O) emulsions. Incorporating a permitted low HLB emulsifier into the oil phase such as sorbitan mono-oleate allowed for a single stage process to create $\mathrm{W} / \mathrm{O} / \mathrm{W}$ emulsions, ${ }^{14}$ however, this is only possible at high emulsifier concentrations, which would be impractical in real systems, and the shear sensitivity is also poor. Using appropriate levels of low HLB emulsifiers usually requires a 2 stage homogenisation process, and in the food sector, the only permitted emulsifier which shows excellent 

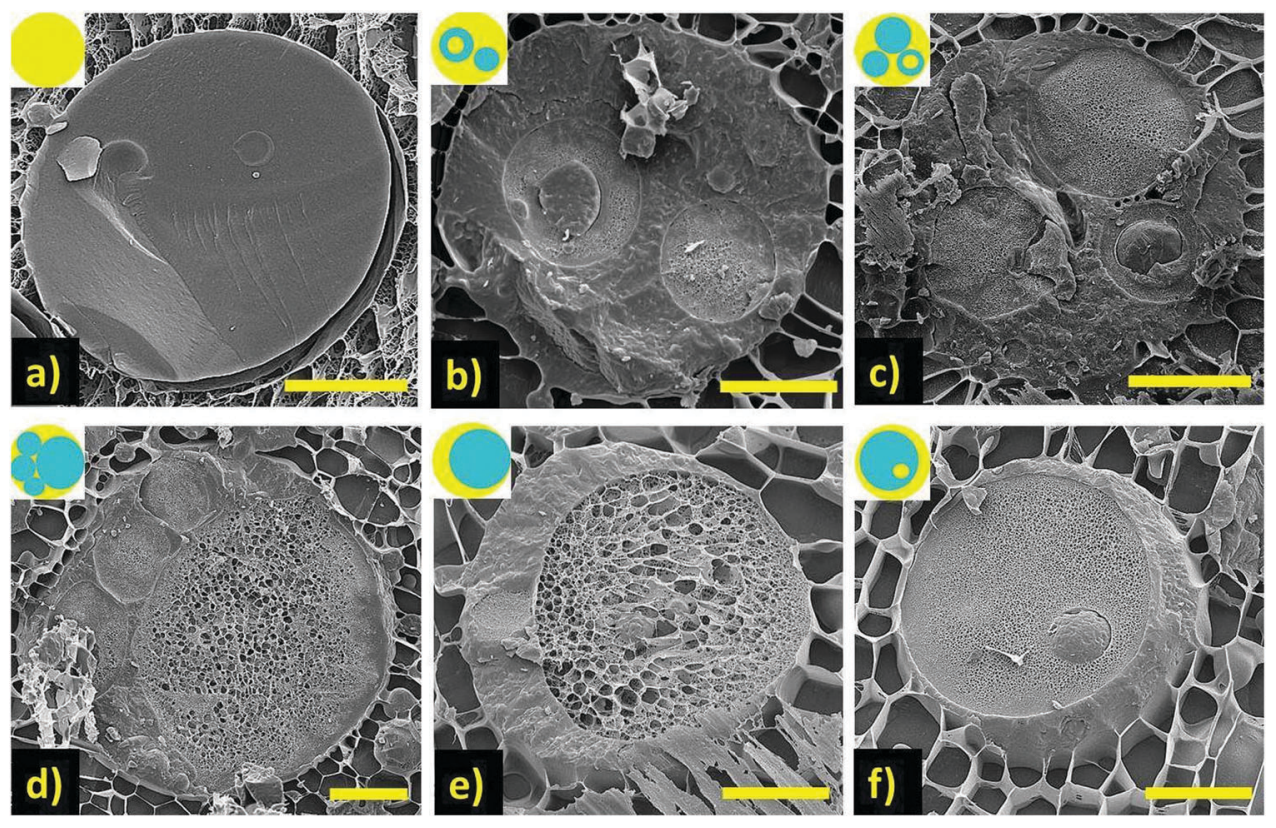

Fig. 2 Representative cryo-SEM images (scale bars $10 \mu \mathrm{m}$ ) of oil/water emulsion samples prepared by harnessing biopolymer phase separation of the water phases with compositions: (a) only LBG; (b) 1 wt\% LBG: Car (1:1); (c) 2 wt\% LBG: Car (1:1); (d) 3 wt\% LBG: Car (1:1) and (e) \& (f) 4 wt\% LBG: Car $(1: 1)$. Insets: drawings to guide the eye, water (blue) and oil (yellow) phase in corresponding images. Reproduced from ref. 15 (DOI: $10.1016 / j . f o o d h y d$. 2014.12.029) under the terms of the Creative Commons Attribution License.

stability is polyglycerol polyricinoleate (PGPR). However, recent work has demonstrated the potential for food permitted emulsifiers to generate single-stage multiple $\mathrm{W} / \mathrm{O} / \mathrm{W}$ emulsions. Patel $e t ~ a l .{ }^{15}$ showed that by inducing phase separation between the two water phases using incompatible biopolymer mixtures, locust bean gum (LBG) and carrageenan (Car), led to the formation of multiple $\mathrm{W} / \mathrm{O} / \mathrm{W}$ emulsions in a single step (Fig. 2). However, the yield and proportion of water encapsulated is as yet not particularly well controlled, but this may well be improved with further development.

Below we review recent work on the fabrication of multiple emulsions using three contrasting approaches: microfluidic production, droplet stabilization using block copolymers and droplet stabilization using colloidal particles. In the first case, exclusive use of microfluidics yields exotic structures in small quantities; however, the field is now moving towards the combined use of microfluidics and phase separation which can potentially solve the bulk production problem. In the second case, the reason for the success of block copolymers in stabilizing multiple emulsions prepared in a single step has just recently been proposed. In the third case, we argue that solvent trapped at particle surfaces or within particle clusters plays a crucial role in multiple emulsion stabilization.

\section{Microfluidic approach}

Multiple emulsions can be generated one droplet at a time within microfluidic devices from well-defined instabilities that are accessed via the tuning of the flow rates and flow patterns of the liquids being emulsified. ${ }^{16-19}$ As a result, emulsion droplets created within microfluidic devices tend to be far more mono-disperse than those created in bulk. Characteristics such as order, size, number and configuration of encapsulated droplets can all be selected. Accordingly, droplets fabricated in this manner can be tailored for application in the active delivery of fragrances, enzymes and the controlled release of pesticides. ${ }^{20,21}$ They can also be designed for fundamental studies of transport processes in multiple emulsion droplets. ${ }^{22}$ A significant drawback is the production efficiency of microfluidics which suffers in comparison to that of bulk formation. ${ }^{17,18}$

The most relevant microfluidic droplet creation geometries here are T-junctions, flow-focusing or co-flow (although other options exist ${ }^{23}$. In the T-junction, the continuous phase is flowing along the top of the $\mathrm{T}$ and the dispersed phase enters via a perpendicular channel (the stem of the T). Droplet formation occurs due to the combined effect of pressure changes in the continuous phase and the squeezing of the dispersed phase. In flow-focusing microfluidics, parallel streams of the dispersed and continuous phases are focused through a constriction. The dispersed phase breaks up into droplets as a consequence of the flow field through the gap. In a co-flowing geometry there are two concentric channels: the dispersed phase is injected centrally into a stream of the continuous phase flowing in the same direction. ${ }^{19}$

Three groups simultaneously pioneered the single-step production of multiple emulsions via microfluidics: the Kumacheva group and the Gañán-Calvo group implemented flow-focusing for several fluid streams, ${ }^{24}$ including the use of a loudspeaker, ${ }^{25,26}$ whilst the Weitz group succeeded by combining co-flow and flow focusing in a single device (Fig. 3a). ${ }^{27}$ In the first two approaches, a coaxial jet made up of the three phases forms as a result of the focusing geometry and breaks up into composite droplets as a consequence of the Rayleigh-Plateau instability. By varying the flow regimes the 
a

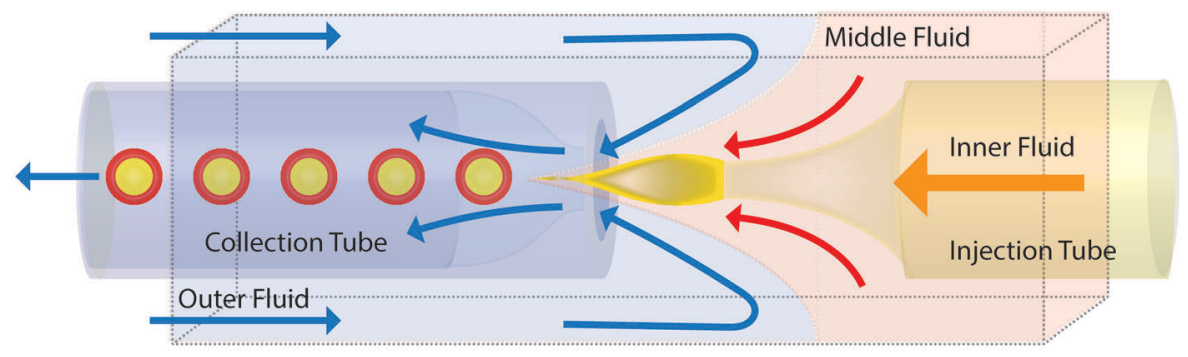

b Ternary Mixture

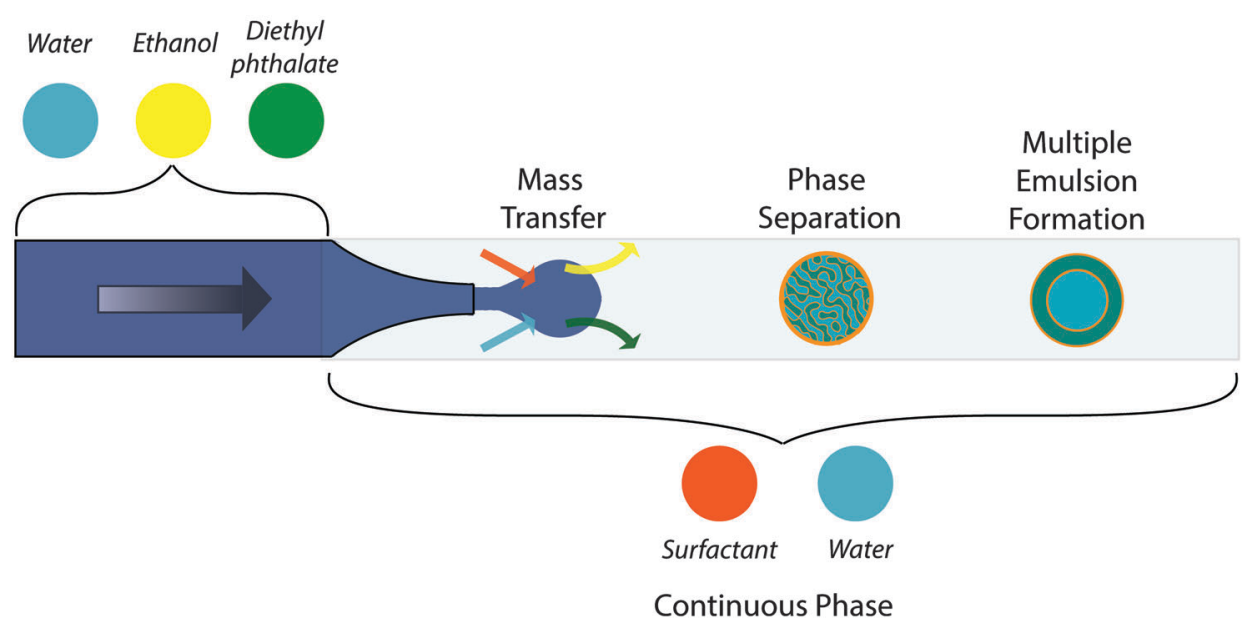

Fig. 3 (a) Schematic showing the microfluidic production of multiple emulsions using combined co-flow and flow focusing in a single device. Adapted from ref. 27. (b) Schematic showing the microfluidic production of multiple emulsions using combining droplet production using flow focusing with phase separation. Based on description in ref. 35.

Kumacheva group was able to control the size of inner and outer droplets and the number of inner droplets. ${ }^{24}$ In the third approach, counter-posed glass capillaries, one tapered (for co-flow) and one cylindrical (for focussing), within a square cross-section surround were used to produce double emulsions, see Fig. 3a. The outer droplets were typically a little over $50 \mu \mathrm{m}$ diameter and there was also good control of the relative size of the inner and outer droplets and the number of inner droplets. ${ }^{27}$

Kim and Weitz were able to go on to generate triple emulsions in a single step by adapting the combined co-flow and flow focusing design. ${ }^{28}$ Specifically, they tapered the capillary which creates the flow focusing constriction so as to accommodate an additional middle fluid. This was used to form $\mathrm{W} / \mathrm{O} / \mathrm{W} / \mathrm{O}$ triple emulsions and by inverting the modification of the glass surface (i.e. hydrophobic surfaces were changed to hydrophilic ones and vice versa) they were also able to fabricate $\mathrm{O} / \mathrm{W} / \mathrm{O} / \mathrm{W}$ emulsions.

The combined co-flow and flow focusing approach ${ }^{27}$ has also been applied to the fabrication of air/oil/water composite droplets. ${ }^{29,30}$ In both cases these were post processed to yield hollow capsules with solid shells.

The various devices outlined above have been employed to create droplets with an amazing level of control. Sophisticated designs have been used to tailor the flow fields. Because of this sophistication, it seems unlikely that this approach will ever yield bulk quantities of multiple emulsion droplets. One solution to this problem is currently growing in popularity: simplified flow conditions can be combined with a phase separation transition to create a diverse range of multicomponent droplets. ${ }^{31-37}$ The phase separation is triggered either by a change in temperature or a change in composition due to one or more components entering or leaving the droplet.

Phase separating liquids have been used previously to create multiple emulsions, albeit via a multi-step route. ${ }^{38-40}$ More recently, multiple emulsions have been made in a single step within microfluidic devices by utilising de-mixing processes. This was first demonstrated by Zhao and Middelberg who created droplets at a T-junction; ${ }^{31}$ the continuous phase was water and the dispersed phase was Miglyol 812 oil, ethanol, and water. Phase separation was driven by the diffusion of ethanol into the continuous phase. Whether the droplet was simple $\mathrm{W} / \mathrm{O}$ or $\mathrm{O} / \mathrm{W} / \mathrm{O}$ could be controlled via the choice of surfactant. The microfluidics behind this work is remarkably simple compared to all the multiple emulsion studies which preceded it. Similar research was carried out more recently with the variation that the phase separation was triggered by a cosolvent moving from the continuous phase into the dispersed phase. ${ }^{34}$ 
In this case the ingredients were chosen for the purpose of polymerizing the complex droplets formed (which included Janus droplets as well as high order multiple emulsions).

One noticeable problem with this mass transfer approach to creating a multiple emulsion is that the transfer becomes unmanageably fast for very small droplets. This leads to phase separation occurring before droplet formation is complete. Jeong et al. developed a 3D flow focusing geometry and paid careful attention to the position of the constriction so as to be able to create well-controlled multiple emulsion droplets of diameter $2 \mu \mathrm{m}$ or less (Fig. $4 \mathrm{a}-\mathrm{d}$ ). ${ }^{32}$ The smallest droplets they fabricated, which may well be multiple emulsions, were diameter $50 \mathrm{~nm}$ ! Relatively small droplets (down to $10 \mu \mathrm{m}$ ) were also prepared by Haase and Brujic who triggered phase separation by the movement of two components out of the droplet while the surfactant diffused in from the continuous phase, see Fig. $3 b .^{35}$ By tuning composition, they were able to select for multiple emulsions with orders between 1 and 5 that held alternating water rich and oil rich compartments. The results could be systematized on a 'phase' diagram of emulsion order as a function of outer drop diameter and starting water content.
Similar phase separation, triggered by a change in composition (or temperature, ${ }^{15}$ as described in the Introduction), is observed for some pairs of polymers dispersed in water. These are often called water/water or all-aqueous emulsions and are particularly advantageous for application in the cosmetic and food industries and can also be used as liquid vessels for protein delivery and microreactors for enzyme reactions. ${ }^{6,33,41}$

Microfluidic emulsification of the precursor polymer dispersions is challenging due to the extremely low interfacial tension. Typically external fields (e.g. mechanical vibration) are needed to drive the creation of droplets. ${ }^{42}$ Multiple emulsions have been prepared in these systems with the phase separation transition driven by the movement of water from inside to outside the droplet. ${ }^{33}$ By tuning the amplitude and rate of the mechanical perturbation, as well as the flow rate of the fluids, it was possible to prepare monodisperse emulsions.

The first study combining microfluidics with molecular phase separation induced by a temperature change was carried out very recently. ${ }^{37}$ (Previously, colloidal-scale phase separation was harnessed to make Janus droplets ${ }^{36}$ ) This approach presents a distinct advantage because the interfacial tensions can be continuously varied even to the extent that the internal

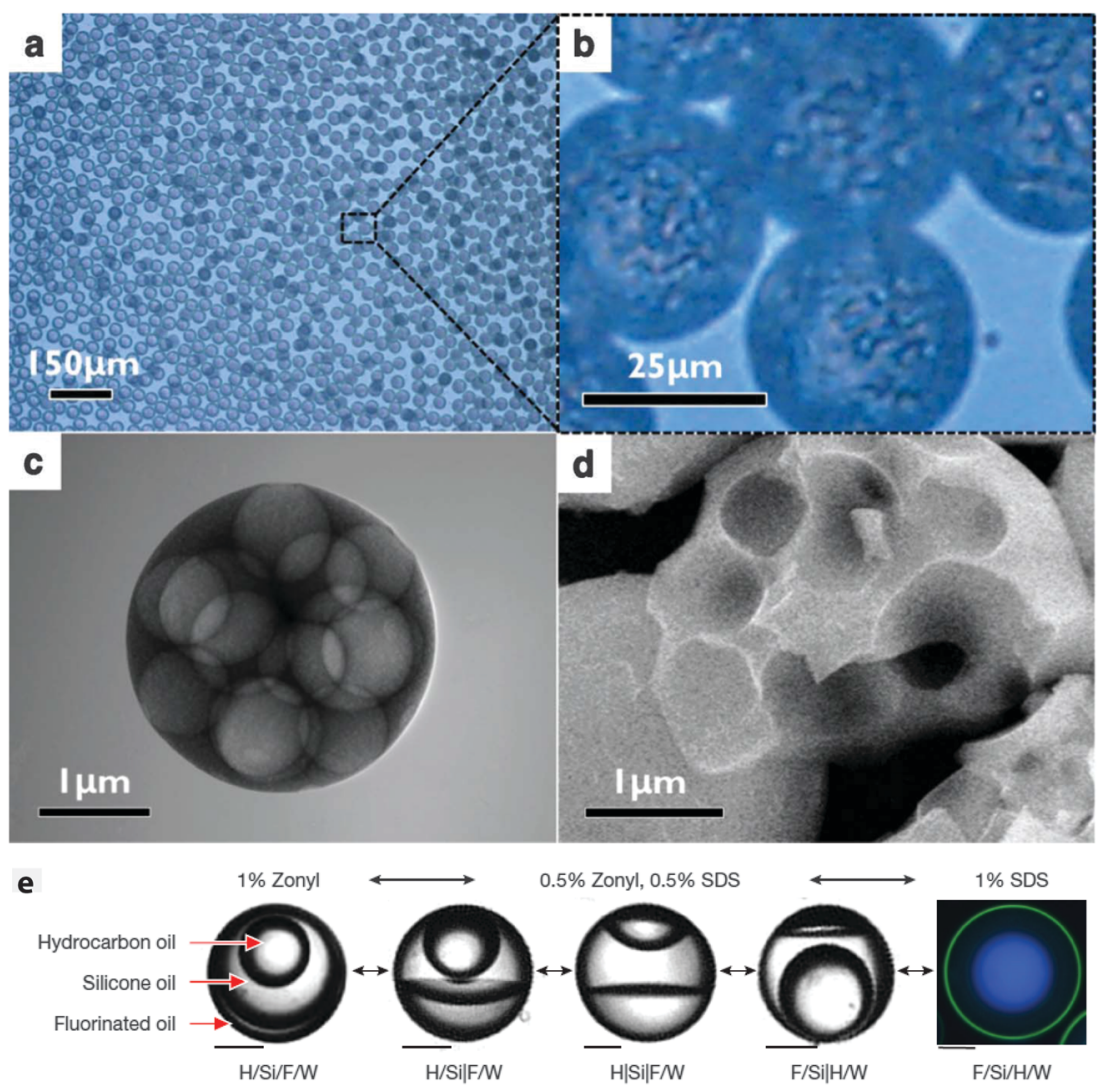

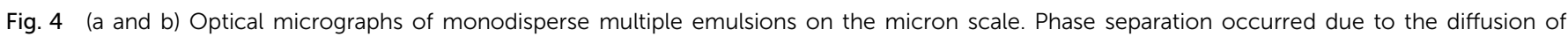

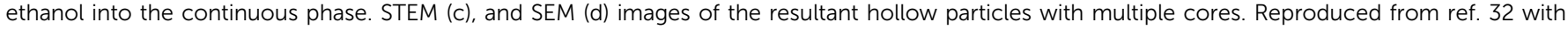

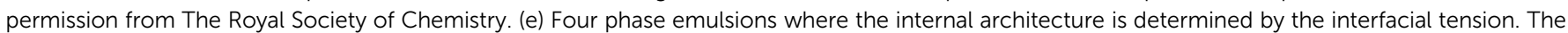

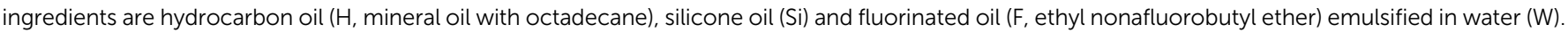

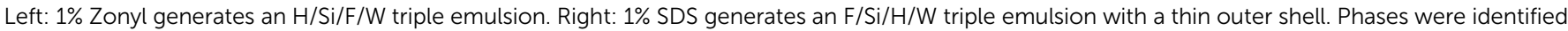

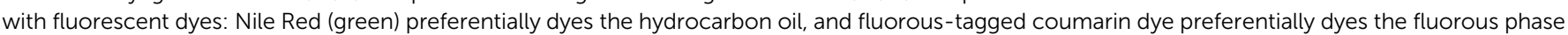
(blue). Scale bars, $50 \mu \mathrm{m}$. Reprinted by permission from Macmillan Publishers Ltd: Nature (ref. 37), copyright (2015). 
phases can be induced to remix. For the molecular study, the authors use a partially miscible fluorocarbon-hydrocarbon pair and develop a quantitative understanding of the surface energies which they deploy to tune the composite droplet structures (Fig. 4e). ${ }^{37}$ Because they understand the relationship between the droplet structure and the surface energies they can induce changes in droplet conformation after formation using stimuli responsive surfactants.

While microfluidic devices are typically only useful for low volume applications, the research described above does point towards a well-controlled route to multiple emulsion formation. The flow fields required to prepare multiple emulsions are typically very simple provided that a phase separation transition is harnessed to produce the internal architecture of the droplets. ${ }^{31-37}$ Such an approach could be implemented on a large scale via, for example, dynamic membrane emulsification. ${ }^{43}$

\section{Block copolymer approach}

While block copolymers themselves are not often used as food ingredients, they are widely used as surfactants for personal care applications $;^{44}$ furthermore, the fundamental soft matter research carried out in this area is instructive. Early work on multiple emulsions involved cross-linking the polymers and/or the combined use of other surfactants. ${ }^{1}$ The first multiple emulsions stabilized by block copolymers alone were prepared from blocks made of different peptides. ${ }^{45}$ The hydrophilic portion of the polymer was made from units of L-lysine whereas the hydrophobic half was made from units of leucine (mixed chirality to give a racemic hydrophobic block). Silicone oil and water were used as the liquids and emulsification was via ultrasound and repeated high pressure homogenization. Multiple emulsions formed $(\mathrm{W} / \mathrm{O} / \mathrm{W})$ with a single internal droplet and outer droplets $\sim 250 \mathrm{~nm}$ in diameter. Zhang et al. subsequently returned to this system ${ }^{46}$ finding that salt plays a curious role: the cation had no influence on the structure of the emulsion formed; however, there were significant changes for multi-charged anions. Chloride gave a single internal droplet, sulphate gave a small number of internal droplets whilst phosphate gave a dense population of internal droplets.

A body of recent research suggests that osmotic pressure can be used to control the one-step formation of multiple emulsions. Two groups (working with non-edible ingredients) have explored the very stable multiple emulsions which form when block copolymers are used to stabilize the interfaces. In 2012, the Ngai group began working with block copolymers of polystyrene and polyethylene glycol. ${ }^{47}$ Extraordinarily, they were able to produce $\mathrm{W} / \mathrm{O} / \mathrm{W}$ emulsions in a single step using toluene as the oil (oil volume fractions $20-70 \%$ ). After two minutes in a rotor stator the multiple emulsion is stable for six months at least, Fig. 5. The authors suggest that their system benefits from both chains being extended and from the fact that the polymers do not form micelles in the oil phase. In a follow up work, this team varied the block sizes of the polymer: for long polystyrene blocks, unsurprisingly, only $\mathrm{W} / \mathrm{O}$ emulsions formed. ${ }^{48}$ The novelty occurred
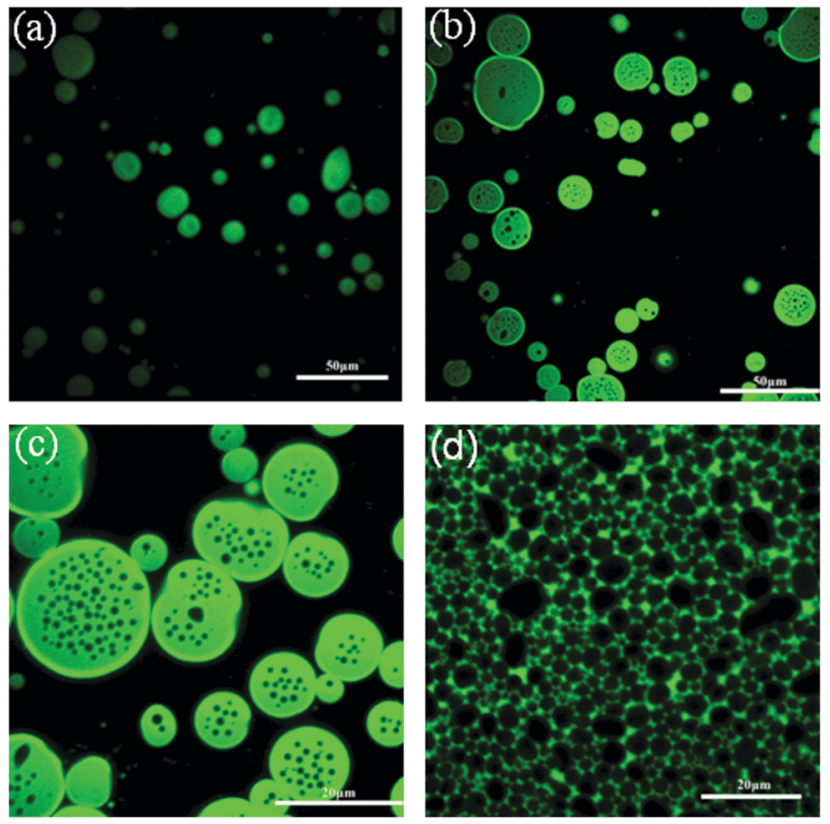

Fig. 5 Confocal microscopy images of copolymer-stabilized emulsions prepared in a single step by shearing a mixture containing toluene, water, and PEG/PS block copolymers at different volume fractions of oil: (a) $15 \%$ toluene, (b) $22 \%$ toluene, (c) $40 \%$ toluene, and (d) $80 \%$ toluene. Reprinted with permission from ref. 47. Copyright (2012) American Chemical Society.

when the polystyrene block was very short: this resulted in a startling disconnection between multiple emulsion formation and emulsion inversion. At low toluene concentrations a $\mathrm{W} / \mathrm{O} / \mathrm{W}$ emulsion forms. As more toluene is added the system switches to forming an $\mathrm{O} / \mathrm{W}$ emulsion then for even more toluene there is a catastrophic inversion to $\mathrm{W} / \mathrm{O}$.

Besnard et al. discovered the same multiple emulsion behaviour almost simultaneously. ${ }^{49}$ This team worked with a polystyrene/ poly(DMAEMA) surfactant; the second block is hydrophilic to an extent which varies with temperature. Water and toluene were again the liquids of choice. They demonstrated stable multiple emulsion formation close to the inversion point when it was induced via temperature or via $\mathrm{pH}$. They suggested that the formation conditions are: that the spontaneous curvature of the interface needs to be reasonably low while the interfacial tension is not too low. In the follow up work, the same team explored a broader spectrum of polymer architectures with a wider range of techniques. ${ }^{50}$ The authors showed that, depending on the polymer, there are two different ways the emulsions invert from $\mathrm{O} / \mathrm{W}$ to $\mathrm{W} / \mathrm{O}$ (with increasing $\mathrm{pH}$ ). Either there is a transitional state which involves both types of emulsion being simultaneously unstable or there is a transitional state which is a $\mathrm{W} / \mathrm{O} / \mathrm{W}$ multiple emulsion (stable and formed in a single step). The authors concluded that stable multiple emulsion formation occurs when the polymer favours a flat compact conformation at the interface with zero spontaneous curvature, but simultaneously shows highly asymmetric partitioning behaviour between the two phases. The polymers which do this are described as exhibiting anti-Bancroft behaviour, because they favour curving away from the water while not partitioning into 
the water. Another curious observation is that for fixed composition the behaviour appears to depend on the molar mass of the polymer. A smaller molecule does not invert/form multiple emulsions whereas a larger one does. The authors also point out that none of their varied molecules is able to stabilize an $\mathrm{O} / \mathrm{W} / \mathrm{O}$ multiple emulsion. They suggest this might be to do with electrostatic interactions being too strong to allow appropriate interfacial curvatures. A third team has made new molecules with the aim of harnessing the same facile emulsification process. ${ }^{51}$ However, overall much remains to be explained.

Recently, Bae et al. have proposed ${ }^{52}$ that the highly stable multiple emulsions stabilized by block copolymers, described above, ${ }^{47-50}$ are related to the presence of electrolyte in the samples. The electrolyte can be a remnant of the catalyst used in the polymer synthesis or alternatively could be added afterwards to give well controlled emulsification performance. The authors suggest that metal ions are present with the polymer in any oil droplets created in an emulsion sample. In this configuration, water will be dragged from the continuous phase into the oil driven by the osmotic pressure, Fig. 6 . The final internal droplets adopt a size which is a balance between the Laplace pressure of the droplets and the osmotic pressure due to the metal ions. They demonstrate that the metal ion concentration that might remain from synthesis is sufficient to drive this process. Furthermore they demonstrate the effect of adding to or subtracting from this reservoir. In line with this picture of multiple emulsion formation, they are able to remove the internal droplets by modifying the osmotic pressure of the continuous phase.

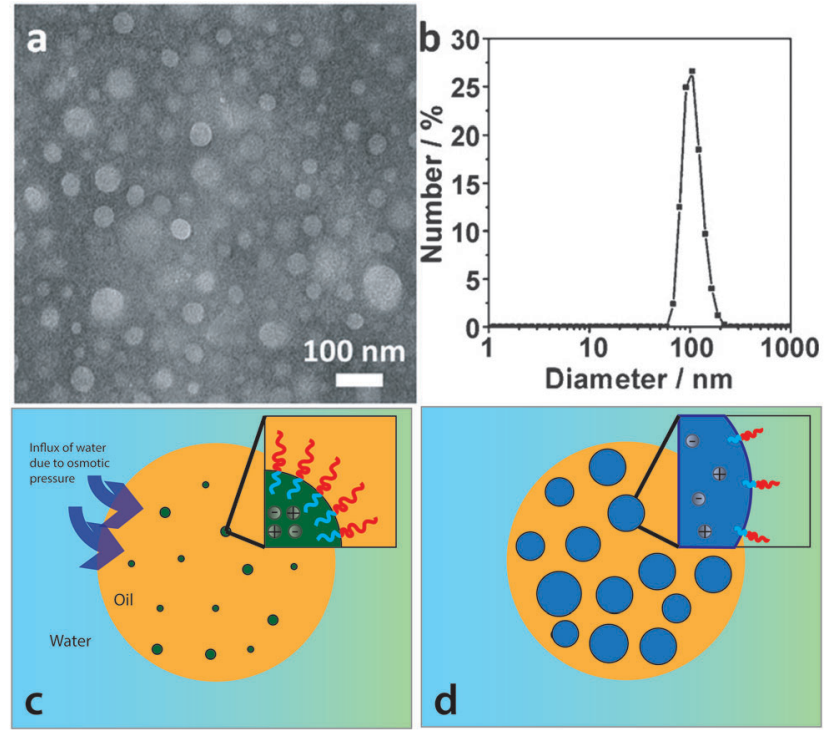

Fig. 6 Proposed mechanism for multiple emulsion formation: (a) transmission electron microscopy image and (b) size distribution of salt aggregates dispersed in the organic solution (chloroform containing $10 \mathrm{mg} \mathrm{mL}^{-1}$ of as received PS-PNIPAM). (c) and (d) Illustration of the spontaneous formation of water droplets at an O/W droplet interface: (c) water is moves into the oil phase due to the presence of salt aggregates, (d) this leads to osmotically driven formation of a W/O/W double emulsion. Images are reproduced/adapted from ref. 52 with permission.
The work of Bae et al. rationalizes the disconnection between emulsion inversion and the single-step formation of multiple emulsions. ${ }^{52}$ It also explains why $\mathrm{O} / \mathrm{W} / \mathrm{O}$ multiple emulsions are not found. Because of the stability of the resulting multiple emulsions and the ease of control of the size of the internal droplets it would be highly desirable to be able to achieve the same behaviour with edible ingredients.

\section{Pickering approach}

Pickering emulsions (droplets stabilized by colloidal particles) have been the subject of intense study since the late 90s although they have been known about for very much longer. ${ }^{53,54}$ When two immiscible solvents and appropriate colloidal particles are mixed together the type of emulsion which forms depends, in part, on the wetting character of the particle surfaces. If the particles are slightly more hydrophilic this will tend to favour the formation of $\mathrm{O} / \mathrm{W}$ emulsions and conversely if the particles are slightly more hydrophobic W/O are more likely to form. Other parameters such as the proportions of the two liquids, processing route, $\mathrm{pH}$, temperature and presence of other interfacially active ingredients also control the type of emulsion formed.

Pickering emulsions can be extremely robust and have been explored in a very wide variety of systems. ${ }^{55-57}$ They have been attracting considerable interest in the food sector due to the added functionalities such as stability, taste, texture etc. However, the main obstacle is finding functional, yet food permitted particulate systems. ${ }^{55}$ Fat crystals have been used to stabilise the primary $\mathrm{W} / \mathrm{O}$ droplets, ${ }^{58,59}$ by crystallising saturated monoglycerides and a saturated triacylglycerol in the oil phase. These crystals form a shell around the water droplets upon cooling conferring high stability, and high encapsulation efficiency. Starch particles, ${ }^{60,61}$ structured cellulose ${ }^{62-64}$ and cocoa particles $^{65}$ amongst others ${ }^{66,67}$ have been employed as edible particulate stabilizers. In multiple emulsions, particles have also been used to stabilise the secondary oil droplets in $\mathrm{W} / \mathrm{O} / \mathrm{W}$ multiple emulsions. ${ }^{68}$ The particles form a mechanical barrier which reduces the loss of the internalised water droplets, thus improving the overall stability of the system. This could also lead to a reduction in emulsifier levels (an attraction in food emulsions). Whether or not these systems are amenable to a one-stage process remains to be seen, nevertheless, combining this approach with that of the phase-separated polymers described above ${ }^{15}$ to instigate a one-stage emulsification process could have potential in food applications.

Early observations of single-step multiple emulsion formation by Binks et al. involved oils that potentially contained several components. ${ }^{69,70}$ The first study deployed partially hydrophobic fumed silica with triglyceride oil; the second used monodisperse silica nanoparticles and PDMS based oil. Multiple emulsion formation was found close to the composition at which inversion occurred (although this depended strongly on which solvent the particles were initially dispersed in). The authors suggest that these systems have two populations of interfacial stabilizers either due to the action of a component of 
the oil phase or due to adsorption onto the particles of a component from the oil phase. By this route, it is proposed that these systems can stabilize two different types of interface. Soon after this the Armes group observed multiple emulsions when polystyrene particles, coated with block copolymer hairs, whose charge state could be varied via the $\mathrm{pH}$, were used to stabilize fluid interfaces. ${ }^{71,72} \mathrm{O} / \mathrm{W} / \mathrm{O}$ emulsions were observed, for example with methyl myristate at $\mathrm{pH} 8.2 .^{71}$ In the second study, inversion from $\mathrm{O} / \mathrm{W}$ to $\mathrm{W} / \mathrm{O}$ occurs with increasing temperature with multiple emulsions forming at intermediate temperatures. ${ }^{72}$ The authors suggest that the temperature increase disrupts the hydrogen bonding around the steric hairs resulting in the particles becoming more hydrophobic. The relationship between multiple emulsion formation and inversion has been further explored recently using more exotic particles. ${ }^{73-75}$

Crucial experiments on particle-stabilized multiple emulsions were performed by Binks et al. as a function of particle concentration. This was first carried out for fumed silica particles with a cyclic silicone oil. $^{76}$ Inversion was observed for particles of intermediate hydrophobicity only if the particles were initially dispersed in the oil phase. Furthermore, O/W emulsions were observed for low concentrations of particles with inversion occurring with increasing particle concentration. This was found for two different particle surface chemistries - but multiple emulsion formation was only observed for the more hydrophilic particles. The authors demonstrated that flocculation is important for this effect by carrying out emulsification using ultrasound. In this case, all of the particles were dispersed and the resulting emulsions are all $\mathrm{O} / \mathrm{W}$. The authors suggest that flocculation causes a change in particle wettability because the flocs use up silanol groups in interparticle bonds. The second study introduced a new route to preparing particle-stabilized emulsions (see Fig. 7a) in an effort to remove the influence of the initial choice of solvent. ${ }^{77}$ This powder approach involves sprinkling the particles onto the more dense solvent before gently pouring the less dense solvent on top. Here again it is found that particle concentration will induce inversion, provided that the particle wettability is already close to that for which inversion occurs. Multiple emulsions could be formed close to inversion and it was suggested that this was again a result of particle clusters behaving as though they were more hydrophobic than dispersed particles. This conjecture was supported by a demonstration of inversion
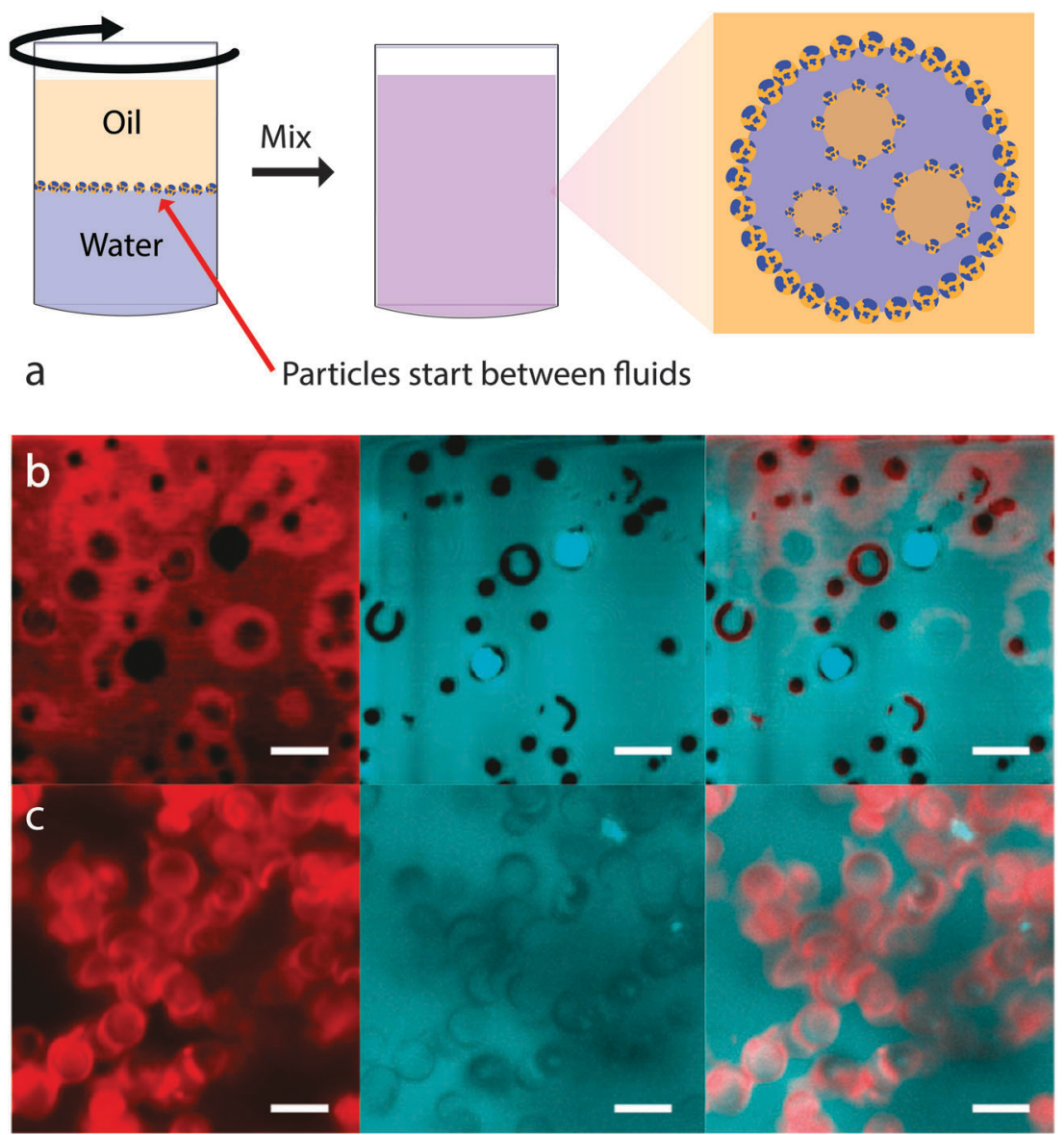

Fig. 7 (a) Placement of particles between fluids prior to preparing a multiple emulsion. Particle wettability seems to be modified by flocculation possibly because the flocs use up silanol groups in interparticle bonds. (b and c) Fluorescence confocal microscope images of microbowl particles: (b) in water and (c) in $n$-dodecane. Before being dispersed in water ( $n$-dodecane), the microbowls were dispersed in $n$-dodecane (water) and filtered through filter paper. Red in the left frames indicates the presence of water; black and blue in the centres of the microbowls (in the centre and right frames) suggests the presence of air and oil respectively. Images (b) and (c) reprinted with permission from ref. 79. Copyright (2011) American Chemical Society. 
from $\mathrm{W} / \mathrm{O}$ via $\mathrm{O} / \mathrm{W} / \mathrm{O}$ to $\mathrm{O} / \mathrm{W}$ as a function of mixing time. With increasing time the particles will have been better dispersed which the authors believe then exposes a greater proportion of silanol groups. Further experiments using the powdered particle technique have been carried out on emulsions where the composition of the aqueous phase was varied using propanediol. ${ }^{78}$

Even more suggestive are the experiments which have been carried out by Nonomura $e t$ al. using silicone particles in the shape of bowls of dimension 2.5 micron, called microbowls. ${ }^{79}$ These particles were used alone or in combination with spherical particles of the same surface chemistry. In these systems multiple emulsion formation was common and was demonstrated with a wide variety of oils. The authors suggest that the distinctive shape of the particles gives rise to dramatic contact angle hysteresis. Multiple emulsions were only observed when the particles are first dispersed in the oil phase, with water subsequently added. The importance of this route was demonstrated using fluorescence imaging, Fig. 7b and c. Particles dispersed in oil and then transferred to water include trapped oil or trapped air "in the bowl". Those first dispersed in water and then transferred to oil are covered in an aqueous surface layer. The authors call this sensitivity to the particle history "imprinting". We suggest this concept could well be applicable beyond bowl shaped particles.

Very recently it has been demonstrated that spikey particles can trap air and hence be transformed from hydrophilic to hydrophobic (or vice versa). ${ }^{80}$ These observations were made in a different context, but they provide some support for the idea that trapping of fluid is responsible for changing the apparent wettability of particles/particle clusters.

White et al. also used adsorption at particle surfaces to control emulsification behaviour albeit at a much more subtle level..$^{81}$ In this case the solvent in question is the physi-adsorbed water that is present at the surface of Stöber silica. Gentle heating can be used to reduce or remove this water layer; whereas, more aggressive heat treatment removes this water and changes the chemical nature of the silica surfaces. The wetting properties of the underlying surface were modified by including amine as well as silanol groups at the particle surfaces. The liquids explored were the partially miscible combination of water and 2,6 dimethyl pyridine (lutidine). Gentle drying removed surface water and made the particles increasingly hydrophobic. $\mathrm{W} / \mathrm{O} / \mathrm{W}$ emulsions were observed when the particles were dried slightly beyond neutral wetting, Fig. 8. In addition to drying, prolonged exposure to the oil phase also displaces the physi-adsorbed water.

As described above, when multiple emulsions were first observed stabilized by a single population of particles it was usually when a multi-component oil was being used. ${ }^{69,70}$ Hence it was suggested that some component in the oil was acting as the second surfactant (or was modifying a fraction of the particle surfaces). Subsequently many more of these systems have been observed and studied, even for the case of single component oils. Hence a new explanation is required based on: (1) multiple emulsions can form in a single step with a single population of particles with a single component oil; (2) the particle surface chemistry needs to be tuned so that the emulsion
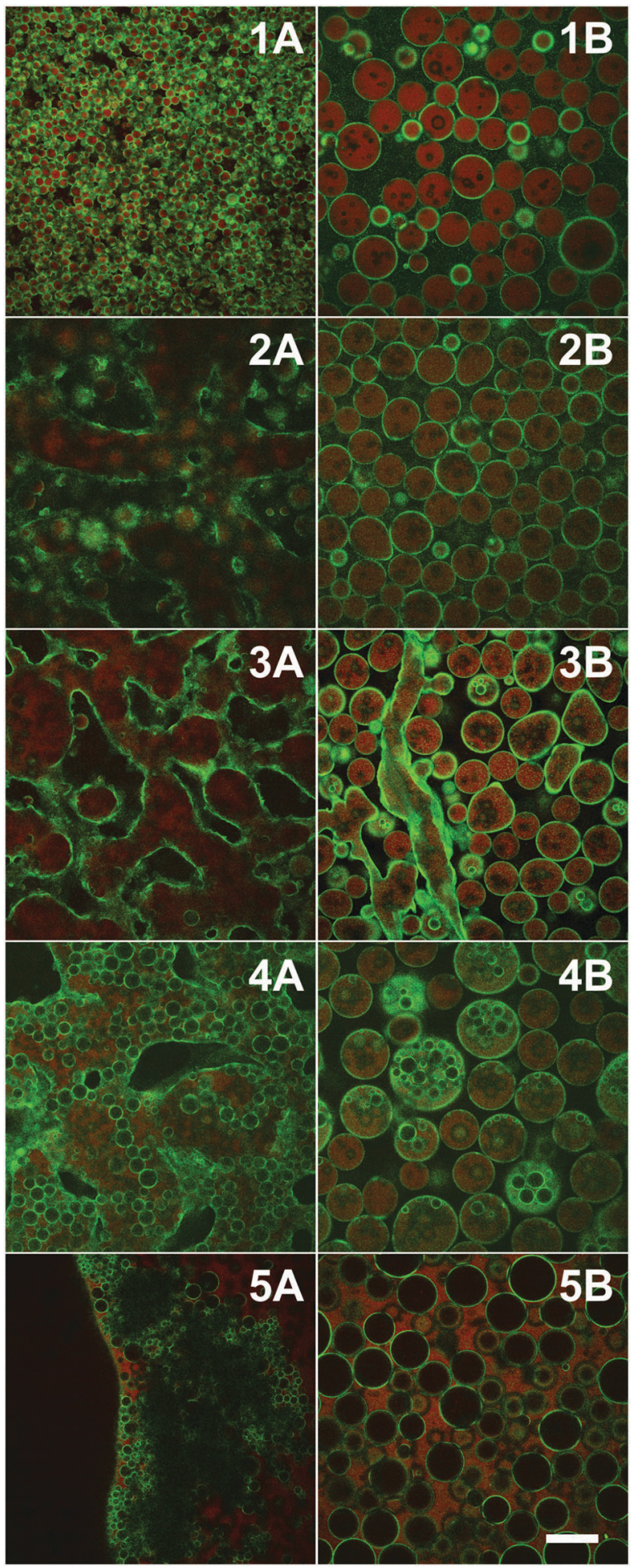

Fig. 8 Confocal microscopy images of emulsions formed by particles dried for increasing lengths of time at $170{ }^{\circ} \mathrm{C}$ (top to bottom 30, 70, 130 , $250 \mathrm{~min}$ and $10 \mathrm{~h}$ ). Left column: emulsions/bijels formed by a phase separation induced by a change in temperature; right column: the same samples after shearing. Gentle drying removed surface water and made the particles increasingly hydrophobic. W/O/W emulsions were observed (4B) when the particles were dried slightly beyond neutral wetting. (Scale bar $=100 \mu \mathrm{m}$; green = silica particles, red = lutidine-rich phase labelled with rhodamine B; sample composition: 28 wt\% lutidine-in-water, 2 vol\% particles; $T=40{ }^{\circ} \mathrm{C}$ ). Reprinted from ref. 81, Copyright (2011), with permission from Elsevier. 
system is close to inversion; (3) multiple emulsion formation is more prevalent at higher particle concentrations where incomplete dispersal has occurred; (4) multiple emulsion formation (often) only occurs after pre-dispersion in oil or using the powder technique and not after pre-dispersion in water; (5) multiple emulsion formation can be controlled by modifying the proportion of adsorbed water on the particle surfaces; (6) multiple emulsion formation is extremely prevalent when microbowls are used as the particles. Based on these observations we suggest that single-step multiple emulsion formation occurs when the particle stabilizers have varied wettability due to adsorbed solvent or trapped air. Microbowls or particle clusters pre-dispersed in an oil phase will trap oil and this will modify their subsequent wetting character, Fig. $7 \mathrm{~b}$ and $\mathrm{c}$. When particle clusters are sprinkled onto a solvent interface they are most likely to trap air (as has been observed with the microbowls). This will also make the clusters/microbowls more hydrophobic. When sufficient energy density is used to disrupt the clusters, multiple emulsions no longer form. In a more subtle way, the wettability of Stöber silica has been modified by changing the adsorption of solvents to yield the same effect, Fig. 8. Providing a mechanism for trapping fluid is crucial to designing a particle for the single-step production of multiple emulsions.

Recently, Tu and Lee have used Janus particles, one styrene face and one acrylic acid face, to create a stable multiple emulsion system. ${ }^{75}$ (The polymer synthesis approach does not use a metal catalyst, unlike the work described in the previous section). Below pH 11 a simple W/O emulsion forms for an oil fraction $>0.5$. Curiously, as further water is added, a stable $\mathrm{W} / \mathrm{O} / \mathrm{W}$ emulsion forms in a single-step process. This unusual behaviour may reflect the different energetics of particles with heterogeneous surfaces. While Janus particles are not made in bulk, these results point towards directions to probe with novel interfacially active proteins.

\section{Conclusions}

We have reviewed three approaches to forming multiple emulsions in a single step: microfluidic production, droplet stabilization using block copolymers and droplet stabilization using colloidal particles. A specific phenomenon is harnessed in each case: phase separation to simplify microfluidics, the electrolyte to drive droplet production in block copolymer systems and the trapping of solvent to create two populations of particles respectively. The fact that some significant level of understanding now exists for these three cases means that multiple emulsions with controlled characteristics can be prepared. We suggest that these systems are now ripe for being exploited. Combining these phenomena with the palette of acceptable ingredients is the remaining challenge.

\section{Acknowledgements}

We are grateful to $M$. Shaver for explaining the basics of block copolymer synthesis, to D. Kraft for pointing out reference 80 and to the BBSRC DRINC scheme (grant BB/I006133/1) for financial support.

\section{References}

1 N. Garti and A. Aserin, Adv. Colloid Interface Sci., 1996, 65, 37-69.

2 N. Garti, Colloids Surf., A, 1997, 123-124, 233-246.

3 G. Muschiolik, Curr. Opin. Colloid Interface Sci., 2007, 12, 213-220.

4 R. Mezzenga, Food Hydrocolloids, 2007, 21, 674-682.

5 L. Sapei, M. A. Naqvi and D. Rousseau, Food Hydrocolloids, 2012, 27, 316-323.

6 A. K. Pawlik, P. J. Fryer and I. T. Norton, in Formulation Engineering of Foods, ed. J. E. Norton, P. J. Fryer and I. T. Norton, Wiley, New Jersey, 2013.

7 F. Jiménez-Colmenero, Food Res. Int., 2013, 52, 64-74.

8 Y. H. Roos, P. J. Fryer, D. Knorr, H. P. Schuchmann, K. Schroën, M. A. I. Schutyser, G. Trystram and E. J. Windhab, Food Eng. Rev., DOI: 10.1007/s12393-015-9125-z.

9 E. Dickinson, Food Biophys., 2011, 6, 1-11.

10 W. Seifriz, J. Chem. Phys., 1925, 29, 738.

11 J. M. Morais, O. D. H. Santos, J. R. L. Nunes, C. F. Zanatta and P. A. Rocha-Filho, J. Dispersion Sci. Technol., 2008, 29, 63-69.

12 J. M. Morais, O. D. H. Santos and S. E. Friberg, J. Dispersion Sci. Technol., 2010, 31, 1019-1026.

13 N. Perez-Moral, S. Watt and P. Wilde, Food Hydrocolloids, 2014, 42, 215-222.

14 M. Cornec, P. J. Wilde, P. A. Gunning, A. R. Mackie, F. A. Husband, M. L. Parker and D. C. Clark, J. Food Sci., 1998, 63, 39-43.

15 A. R. Patel, P. Dumlu, L. Vermier, B. Lewille, A. Lesaffer and K. Dewettinck, Food Hydrocolloids, 2015, 46, 84-92.

16 S. Okushima, T. Nisisako, T. Torii and T. Higuchi, Langmuir, 2004, 20, 9905-9908.

17 L. Y. Chu, A. S. Utada, R. K. Shah, J.-W. Kim and D. A. Weitz, Angew. Chem., Int. Ed., 2007, 46, 8970-8974.

18 R. K. Shah, H. C. Shum, A. C. Rowat, D. Lee, J. J. Agresti, A. S. Utada, L.-Y. Chu, J.-W. Kim, A. Fernandez-Nieves, C. J. Martinez and D. A. Weitz, Mater. Today, 2008, 11, 19-27.

19 C.-H. Choi, J. Kim, J.-O. Nam, S.-M. Kang, S.-G. Jeong and C.-S. Lee, ChemPhysChem, 2014, 15, 21-29.

20 M. H. Lee, S.-G. Oh, S.-K. Moon and S.-Y. Bae, J. Colloid Interface Sci., 2001, 240, 83-89.

21 M. S. Gamal, M. M. El Shafei, H. A. E. Attia and T. G. M. Mohammed, Ind. Crops Prod., 2009, 31, 99-106.

22 J. Bahtz, D. Z. Gunes, E. Hughes, L. Pokorny, F. Riesch, A. Surbe, P. Fischer and E. J. Windhab, Langmuir, 2015, 31, 5265-5273.

23 Emulsion Formation and Stability, ed. T. F. Tadros, John Wiley \& Sons, Weinheim, 2013.

24 Z. Nie, S. Xu, M. Seo, P. C. Lewis and E. Kumacheva, J. Am. Chem. Soc., 2005, 127, 8058-8063.

25 R. Bocanegra, J. L. Sampedro, A. Gañán-Calvo and M. Marquez, J. Microencapsulation, 2005, 22, 745-759.

26 L. Martín-Banderas, M. Flores-Mosquera, P. Riesco-Chueca, A. Rodríguez-Gil, Á. Cebolla, S. Chávez and A. M. GañánCalvo, Small, 2005, 1, 688-692. 
27 A. S. Utada, E. Lorenceau, D. R. Link, P. D. Kaplan, H. A. Stone and D. A. Weitz, Science, 2005, 308, 539-541.

28 S. H. Kim and D. A. Weitz, Angew. Chem., Int. Ed., 2011, 123, 8890-8893.

29 M. H. Lee, V. Prasad and D. Lee, Langmuir, 2010, 26, 2227-2230.

30 R. Chen, P.-F. Dong, J.-H. Xu, Y.-D. Wang and G. S. Luo, Lab Chip, 2012, 12, 3858-3860.

31 C.-X. Zhao and A. P. J. Middelberg, Angew. Chem., Int. Ed., 2009, 48, 7208-7211.

32 W.-C. Jeong, M. Choi, C. H. Lim and S.-M. Yang, Lab Chip, 2012, 12, 5262-5271.

33 Y. Song and H. C. Shum, Langmuir, 2012, 28, 12054-12059.

34 C.-H. Choi, D. A. Weitz and C. S. Lee, Adv. Mater., 2013, 25, 2536-2541.

35 M. F. Haase and J. Brujic, Angew. Chem., Int. Ed., 2014, 53, 11793-11797.

36 R. K. Shah, J.-W. Kim and D. A. Weitz, Adv. Mater., 2009, 21, 1949-1953.

37 L. D. Zarzar, V. Sresht, E. M. Sletten, J. A. Kalow, D. Blankschtein and T. M. Swager, Nature, 2015, 518, 520-524.

38 M. S. Long, C. D. Jones, M. R. Helfrich, L. K. MangeneySlavin and C. D. Keating, Proc. Natl. Acad. Sci. U. S. A., 2005, 102, 5920-5925.

39 H. J. Kim, E. A. Decker and D. J. McClements, Food Hydrocolloids, 2006, 20, 586-595.

40 J. W. Tavacoli, J. H. J. Thijssen, A. B. Schofield and P. S. Clegg, Adv. Funct. Mater., 2011, 21, 2020-2027.

41 V. Tolstoguzov, Biotechnol. Adv., 2006, 24, 626-628.

42 Y. Song, A. Sauret and H. C. Shum, Biomicrofluidics, 2013, 7, 061301.

43 S. Holzapfel, E. Rondeau, P. Mühlich and E. J. Windhab, Chem. Eng. Technol., 2013, 36, 1785-1794.

44 T. Tadros, J. Cosmet. Sci., 2006, 57, 153-169.

45 J. A. Hanson, C. B. Chang, S. M. Graves, Z. B. Li, T. G. Mason and T. J. Deming, Nature, 2008, 455, 85-88.

46 Y. Zhang, J. Gou, F. Sun, S. Geng, X. Hu, K. Zhang, X. Lin, W. Xiao and X. Tang, Colloids Surf., B, 2014, 122, 368-374.

47 L. Hong, G. Sun, J. Cai and T. Ngai, Langmuir, 2012, 28, 2332-2336.

48 G. Sun, M. Liu, X. Zhou, L. Hong and T. Ngai, Colloids Surf., A, 2014, 454, 16-22.

49 L. Besnard, F. Marchal, J. F. Paredes, J. Daillant, N. Pantoustier, P. Perrin and P. Guenoun, Adv. Mater., 2013, 25, 2844-2848.

50 L. Besnard, M. Protat, F. Malloggi, J. Daillant, F. Cousin, N. Pantoustier, P. Guenoun and P. Perrin, Soft Matter, 2014, 10, 7073-7087.

51 X. Huang, R. Fang, D. Wang, J. Wang, H. Xu, Y. Wang and X. Zhang, Small, 2015, 11, 1537-1541.

52 J. Bae, T. P. Russell and R. C. Hayward, Angew. Chem., Int. Ed., 2014, 53, 8240-8245.

53 B. P. Binks and T. S. Horozov, Colloidal Particles at Liquid Interfaces, CUP, Cambridge, 2006.

54 T. Ngai and S. A. F. Bon, Particle-Stabilized Emulsions and Colloids: Formation and Applications, RSC, Cambridge, 2015.
55 E. Dickinson, Curr. Opin. Colloid Interface Sci., 2010, 15, 40-49.

56 A. Yusoff and B. S. Murray, Food Hydrocolloids, 2011, 25, 42-55.

57 S. Lam, K. P. Velikov and O. D. Velev, Curr. Opin. Colloid Interface Sci., 2014, 19, 490-500.

58 N. Garti, A. Aserin, I. Tiunov and H. Binyamin, J. Am. Oil Chem. Soc., 1999, 76, 383-389.

59 S. Frasch-Melnik, F. Spyropoulos and I. T. Norton, J. Colloid Interface Sci., 2010, 350, 178-185.

60 Y. Tan, K. Xu, C. Niu, C. Liu, Y. Li, P. Wang and B. P. Binks, Food Hydrocolloids, 2014, 36, 70-75.

61 X. Song, Y. Pei, M. Qiao, F. Ma, H. Ren and Q. Zhao, Food Hydrocolloids, 2015, 45, 256-263.

62 I. Kalashnikova, H. Bizot, B. Cathala and I. Capron, Langmuir, 2011, 27, 7471-7479.

63 T. Winuprasith and M. Suphantharika, Food Hydrocolloids, 2015, 43, 690-699.

64 X. Jia, R. Xu, W. Shen, M. Xie, M. Abid, S. Jabbar, P. Wang, X. Zeng and T. Wu, Food Hydrocolloids, 2015, 43, 275-282.

65 J. Gould, J. Vieira and B. Wolf, Food Funct., 2013, 4, 1369-1375.

66 E. Dickinson, Trends Food Sci. Technol., 2012, 24, 4-12.

67 M. Rayner, D. Marku, M. Eriksson, M. Sjöö, P. Dejmek and M. Wahlgren, Colloids Surf., A, 2014, 458, 48-62.

68 M. Matos, A. Timgren, M. Sjoo, P. Dejmek and M. Rayner, Colloids Surf., A, 2013, 423, 147-153.

69 B. P. Binks and J. A. Rodrigues, Langmuir, 2003, 19, 4905-4912.

70 B. P. Binks and C. P. Whitby, Langmuir, 2004, 20, 1130-1137.

71 E. S. Read, S. Fujii, J. I. Amalvy, D. P. Randall and S. P. Armes, Langmuir, 2004, 20, 7422-7429.

72 B. P. Binks, R. Murakami, S. P. Armes and S. Fujii, Angew. Chem., Int. Ed., 2005, 44, 4795-4798.

73 Y. He, F. Wu, X. Sun, R. Li, Y. Guo, C. Li, L. Zhang, F. Xing, W. Wang and J. Gao, ACS Appl. Mater. Interfaces, 2013, 5, 4843-4855.

74 M. Tang, T. Wu, X. Xu, L. Zhang and F. Wu, Mater. Res. Bull., 2014, 60, 118-129.

75 F. Tu and D. Lee, Chem. Commun., 2014, 50, 15549-15552.

76 B. P. Binks, J. Philip and J. A. Rodrigues, Langmuir, 2005, 21, 3296-3302.

77 B. P. Binks, P. D. I. Fletcher, B. L. Holt, P. Beaussoubre and K. Wong, Phys. Chem. Chem. Phys., 2010, 12, 11954-11966.

78 B. P. Binks, P. D. I. Fletcher, M. A. Thompson and R. P. Elliot, Langmuir, 2013, 29, 5723-5733.

79 Y. Nonomura, N. Kobayashi and N. Nakagawa, Langmuir, 2011, 27, 4557-4562.

80 J. H. Bahng, B. Yeom, Y. Wang, S. O. Tung, J. D. Hoff and N. Kotov, Nature, 2015, 517, 596-599.

81 K. A. White, A. B. Schofield, P. Wormwald, J. W. Tavacoli, B. P. Binks and P. S. Clegg, J. Colloid Interface Sci., 2011, 359, 126-135.

82 A. R. Abate, J. Thiele and D. A. Weitz, Lab Chip, 2011, 11, 253-258. 